\title{
Statistik und die Kunst des Zählens
}

\author{
Reinhard Viertl \\ Institut für Statistik, Wahrscheinlichkeitsth. u. Versicherungsmath. \\ Technische Universität Wien
}

Der vielzitierte Beginn des dritten Jahrtausends und die damit verbundene Unkenntnis veranlassen mich, diese Bemerkung zu schreiben.

Daß Künstler und andere Personen, die im Umgang mit Zahlen ungenau sind - allerdings sind sie das zumeist nicht, wenn es um Geld geht - hier Probleme mit den wahren Verhältnissen haben, versteht man vielleicht, anders ist es allerdings mit jenen Personen, die beruflich exakt zählen sollten, und dazu gehören auch Statistiker.

Da ein Jahrtausend genau 1000 Jahre hat und nicht 999, ist das letzte Jahr des ersten Jahrtausends unserer Zeitrechnung das Jahr 1000. Das letzte Jahr des zweiten Jahrtausend somit das Jahr 2000. Daher fängt das dritte Jahrtausend am 1. Jänner 2001 an. Folglich ist die Wende zum dritten Jahrtausend zu Silvester des Jahres 2000.

Daß viele Amtsträger Silvester des Jahres 1999 als Schwelle zum dritten Jahrtausend ansehen, liegt wohl am - den Statistikern bekannten - Phänomen der Jubiläumszahlen, also von runden Zahlen, wie es die Zahl 2000 ist. Man wird vermutlich weltweit zu Silvester 1999 viele große Feste feiern, was aber nichts an der Tatsache ändert, dass die Jahrtausendwende ein Jahr später ist.

Die Statistiker werden also noch mindestens bis Ende des Jahres 2000 zuwarten müssen, bis sie die statistischen Daten des laufenden zweiten Jahrtausends erhalten. Und die vielen angeblich letzten Ereignisse in diesem Jahrtausend werden eben nicht die letzten gewesen sein.

Adresse des Autors:

O.Univ.-Prof. Dipl.-Ing. Dr.techn. Reinhard Viertl

Institut für Statistik, Wahrscheinlichkeitstheorie und Versicherungsmathematik

Technische Universität Wien

Wiedner Hauptstraße 8-10

A-1040 Wien

Tel. (+43 1) $58801-10720$

Fax (+43 1) 58801 - 10799

E-Mail: R.Viertl@tuwien.ac.at 